\title{
A case report on ruptured interstitial ectopic pregnancy: diagnostic dilemma
}

\author{
Suman S. Sharma*, A. V. Gokhale, Shonali Agrawal
}

Department of Obstetrics and Gynecology, SSG Hospital, Baroda Medical College, Baroda, Gujarat, India

Received: 29 March 2019

Accepted: 06 May 2019

\section{*Correspondence:}

Dr. Suman S. Sharma,

E-mail: sharmasuman29@gmail.com

Copyright: () the author(s), publisher and licensee Medip Academy. This is an open-access article distributed under the terms of the Creative Commons Attribution Non-Commercial License, which permits unrestricted non-commercial use, distribution, and reproduction in any medium, provided the original work is properly cited.

\begin{abstract}
The ectopic pregnancy occurs when implantation of the blastocyst takes place in a site other than the endometrium of the uterine cavity. Interstitial ectopic pregnancies are gestations that implant within the proximal, intramural portion of the fallopian tubes with high vascularity. Ectopic pregnancy in the interstitial part of the fallopian tubes can be lifethreatening considering the thin myometrial tissue surrounding the gestational sac and high vascularization which may result in catastrophic haemorrhage when interstitium is ruptured. Being a hazardous type of ectopic pregnancy, it becomes extremely important to diagnose and manage it. This condition presents a challenge for clinical as well as radiological diagnosis. Generally, a case of interstitial ectopic pregnancy has typical radiological features distinguishing it from other ectopic. The ultrasonographic finding of interstitial line has better sensitivity (80\%) and specificity (98\%) than eccentric gestational sac location (sensitivity, 40\%; specificity, 88\%) and myometrial thinning (sensitivity, 40\%; specificity, 93\%) for the diagnosis of interstitial ectopic pregnancy. Ultrasound is the mainstay of diagnosis, but magnetic resonance imaging can be helpful in unusual or complicated cases. Interstitial ectopic pregnancy rupturing into the leaves of broad ligament and creating a tamponade effect to alter the clinical presentation is a rare event which presents as a diagnostic challenge. Here authors present a case of ruptured interstitial ectopic pregnancy confined to the leaves of broad ligament, with atypical presentation and radiological features which led to difficulty in diagnosing the interstitial ectopic pregnancy. After laparoscopic confirmation of ruptured interstitial ectopic pregnancy, the patient was managed successfully by laparoscopic cornual resection.
\end{abstract}

Keywords: Broad ligament, Ectopic Pregnancy, Interstitial ectopic, Ultrasonography

\section{INTRODUCTION}

Ectopic pregnancy accounts for approximately $2 \%$ of all pregnancies and is the most common cause of pregnancyrelated mortality in the first trimester. ${ }^{1}$ Ectopic pregnancy occurs when implantation of the blastocyst takes place in a site other than the endometrium of the uterine cavity. Uncommon implantation sites of ectopic pregnancy include the cervix, interstitial segment of the fallopian tube, scar from a prior caesarean delivery, uterine myometrium, ovary, and peritoneal cavity. ${ }^{2}$ Interstitial ectopic pregnancies are gestations that implant within the proximal, intramural portion of the fallopian tubes. These account for only between $1-4 \%$ of all ectopic pregnancies. ${ }^{3}$ It is one of the most hazardous types with mortality rates as high as $2.5 \%$. A history of pelvic pain along with an abnormal $\beta$ human chorionic gonadotropin level should trigger an evaluation for an ectopic pregnancy. Initial evaluation consists of hormonal assays and pelvic ultrasonography. Although ectopic pregnancy is usually diagnosed on the basis of a combination of clinical, laboratory, and ultrasonographic findings, it occasionally is initially identified at MR imaging. ${ }^{4}$ Historically, interstitial pregnancy was considered safe to manage conservatively until over 12 weeks because of the delayed risk of rupture as a result of the protection 
offered by the greater distensibility of the myometrium which covers the interstitial fallopian tube segment. However, over the last decade evidence now suggests that early rupture is not uncommon. ${ }^{5}$ For patients who are medically unstable or experiencing life-threatening haemorrhage, a surgical approach is indicated. For others, management should be based on patient preference after discussion of the risks, benefits, and monitoring requirements of all approaches. ${ }^{6}$ Here, authors describe an unusual case of rupture of interstitial ectopic pregnancy into the leaves of broad ligament with atypical presentation and atypical radiological features which led to the diagnostic dilemma. Later, the patient was diagnosed and managed successfully by laparoscopic intervention.

\section{CASE REPORT}

A 30-year-old woman, gravida 5, para 3, abortion 1 and live 3 , admitted to our hospital for mild lower abdominal pain for 1 week, with history of amenorrhea of two and half months. She had a past history of bilateral laparoscopic tubal ligation done 5 years back. The urine pregnancy test done was positive. On examination, patient was vitally stable, per abdomen was soft, on per speculum cervical os was closed and of multiparous type, pervaginum uterus was bulky, anteverted, bilateral fornices were free and non-tender. The abdominal and pelvis ultrasonography reported irregular hypoechoic area of $51 \mathrm{~mm} \times 51 \mathrm{~mm}$ with multiple variable sized cystic spaces in right cornual region suggestive of ?trophoblastic disease ??A-V malformation possibility of pelvic varices, with endometrial thickness of $7.9 \mathrm{~mm}$. The quantitative assay of beta human chorionic gonadotropin (beta HCG) was $31,438 \mathrm{mIU} / \mathrm{ml}$ on admission. Magnetic resonance imaging of pelvis showed focal, fairly large, irregular multilobulated, irregular shaped, abnormal intensity lesion of $4 \times 3.7 \times 5 \mathrm{~cm}$ in size in right cornua with adnexal extension without intrauterine extension. It showed central cystic areas with peripheral thick irregular nodular wall, with evidence of prominent dilated vessels with flow voids seen in parametrium and myometrium of fundus and combined endometrial thickness measured $8 \mathrm{~mm}$ suggesting possibility of ectopic pregnancy with or without gestational trophoblastic disease or ovarian mass with paraneoplastic syndrome.

In view of positive urine pregnancy test, a provisional diagnosis of interstitial ectopic pregnancy was made, and diagnostic and operative laparoscopy was planned. On laparoscopy, uterus was normal in size. At right cornua, a cystic bluish lesion measuring $3 \mathrm{~cm}$ x $5 \mathrm{~cm}$ was noted extending down in the adjacent right parametrium (as shown in Figure 1). Both tubes with tubal ring, both ovaries and adnexa were apparently normal in appearance. A diagnosis of ruptured interstitial ectopic pregnancy confined to the leaves of broad ligament was made. Decision of excision and evacuation of the products of conception was made. An incision was made over the most prominent area of the lesion, and dark reddish-brown tissue suggestive of products of conception was noted. The product of conception was removed (as shown in Figure 2). Haemostasis was successfully secured using bipolar and blood was transfused as per requirement. The product of conception was removed and sent for histopathological examination which revealed chorionic villi, trophoblastic cells and decidual tissue suggestive of ectopic pregnancy.

No subsequent uterine bleeding was noted. The patient was discharged on 8th postoperative day after an uneventful recovery.

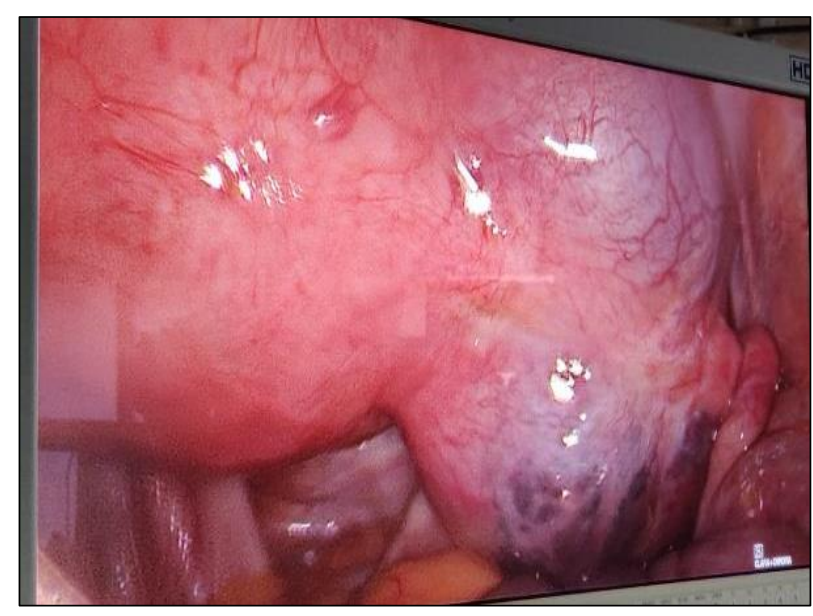

Figure 1: Pseudo-ruptured interstitial ectopic.

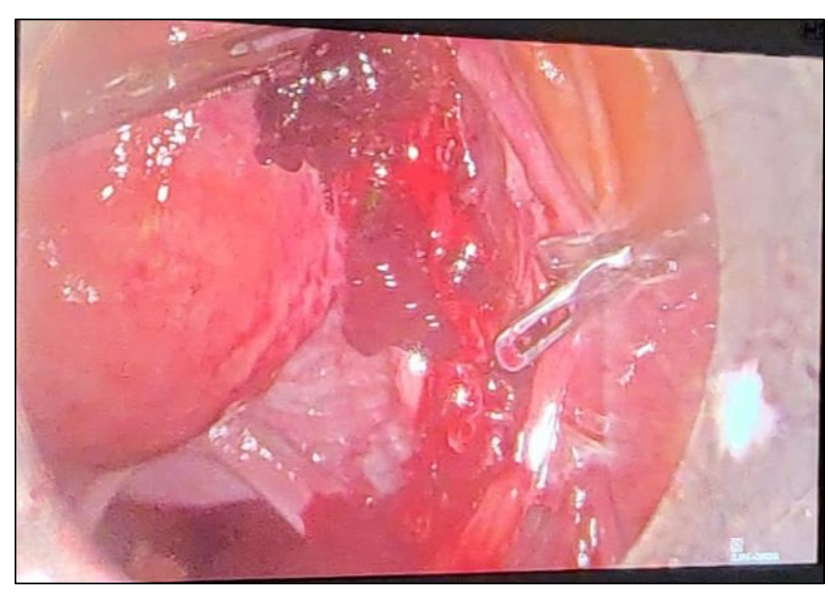

Figure 2: Laparoscopic cornual resection.

\section{DISCUSSION}

Anatomically, the interstitial ectopic pregnancy is located in the intramural portion of the tube covered by myometrium, theoretically allowing a substantial degree of distensibility. Thus, in contrast to a tubal ectopic pregnancy, which may rupture at 6-8 weeks of gestation, an interstitial ectopic pregnancy may progress without symptoms until rupture occurs at 12-16 weeks. Rupture of these pregnancies is associated with high mortality rate as it is situated in proximity with uterine and ovarian arteries which predisposes it to high risk of haemorrhage. 
Early management of these cases prevents the fatal complications. Diagnosis of interstitial pregnancy is challenging but critical to facilitate prompt and appropriate intervention. ${ }^{7}$

The radiological criteria that aid in diagnosis of interstitial tubal ectopic include:

\section{- An empty uterus}

- A gestational sac seen separate from the endometrium and $>1 \mathrm{~cm}$ away from the most lateral edge of the uterine cavity

- A thin $<5 \mathrm{~mm}$ myometrial mantle surrounding the sac.

Apart from the above-mentioned criteria, a specific diagnostic sign, 'The interstitial line sign', which is an echogenic line extending from the gestational sac to the endometrial cavity is of importance. It has better sensitivity (80\%) and specificity (98\%) than eccentric gestational sac location (sensitivity, 40\%; specificity, $88 \%$ ) and myometrial thinning (sensitivity, 40\%; specificity, 93\%) for the diagnosis of interstitial ectopic pregnancy. ${ }^{8}$ These radiological features can be manifested in an unruptured interstitial ectopic but in case of a ruptured ectopic, the features may be atypical and thus it is imperative that the radiologist should be familiar with the variable appearance of ectopic pregnancy at MR imaging. ${ }^{4}$ MRI is applied in cases where more information is needed to assist in diagnosis provided that the patient is hemodynamically stable to undergo the investigation.

In the present case, amenorrhea of two and a half months and a positive urine pregnancy test raised a suspicion of pregnancy. Whereas, the quantitative assay of beta human chorionic gonadotropin (beta HCG) did not correlate with the weeks of gestation and radiological investigation showed the evidence of multiple dilated tortuous vessels with flow voids in parametrium and endometrium on ultrasonography, suggesting a possibility of arterio-venous malformation of pelvic varices. On magnetic resonance imaging, there was a possibility of arterio-venous malformation or an ectopic pregnancy with or without gestational trophoblastic disease or ovarian mass with paraneoplastic syndrome at the right cornual region. In context of the positive findings of pregnancy, an empty uterine cavity with endometrial thickness of $8 \mathrm{~mm}$ and MRI giving a possibility of ectopic pregnancy, a provisional diagnosis of interstitial ectopic gestation was made which was later confirmed as ruptured interstitial ectopic pregnancy on laparoscopy. In spite of rupture of interstitial ectopic pregnancy, the patient was hemodynamically stable with atypical presentation, probably due to the confinement of products of conception and haemorrhage into the broad ligament, creating a pseudo-rupture condition thereby maintaining a tamponade effect. The patient was then managed successfully by operative laparoscopic intervention.
Management of ectopic pregnancy depends on the hemodynamic condition of the patient and level of beta human chorionic gonadotropin (beta-HCG). Earlier detection of ectopic pregnancy allows the patient and physician the option of conservative management. ${ }^{9}$ In the last three decades, systemic methotrexate (MTX) has become widely accepted as the primary treatment for unruptured tubal pregnancy. ${ }^{10}$ However, due to less incidence, consensus regarding methotrexate route and regimen is lacking.

The surgical management includes cornual resection or hysterectomy via laparotomy or laparoscopy. Cornual resection involves removal of products of conception and surrounding corneal myometrium by means of wedge excision. However, increasingly, interstitial pregnancies are treated with laparoscopic cornuotomy, i.e., removal of products of conception with preservation of uterine architecture. Although this technique may increase the incidence of persistent and recurrent interstitial pregnancy, it can potentially maintain patient fertility and decrease their risk for future uterine rupture. ${ }^{11}$

Being at a site of rich vascular supply, measures should be taken to minimize the blood loss intraoperatively. To achieve this, intramyometrial vasopressin used intraoperatively has an extremely important role.

Successful minimally invasive treatment of interstitial pregnancies may be possible in certain cases. Though, collaboration between different specialties continues to be important for improving minimally invasive options. ${ }^{12}$ Patient having high level of beta HCG needs a long surveillance to rule out trophoblastic remnants.

\section{CONCLUSION}

Cornual or interstitial pregnancy with high risk for mortality needs to be identified and treated early to prevent fatal complications. Use of different imaging modalities should be applied to confirm the diagnosis. Early and unruptured cases with low level of beta HCG can be managed conservatively. Ruptured and unstable patients should be managed by appropriate surgical measures. The risk of uterine rupture in subsequent pregnancy is same with medical or surgical management. Thus, careful observation of these women during pregnancy, along with strong consideration of elective caesarean delivery, is warranted in future pregnancy.

\section{ACKNOWLEDGMENTS}

Authors would like to thank the respective professors, seniors and colleagues who guided me through this work.

\author{
Funding: No funding sources \\ Conflict of interest: None declared \\ Ethical approval: Not require
}




\section{REFERENCES}

1. Lin EP, Bhatt S, Dogra VS. Diagnostic clues to ectopic pregnancy. Radiographics. 2008;28(6):166171.

2. Chukus A, Tirada N, Restrepo R, Reddy NI. Uncommon implantation sites of ectopic pregnancy: thinking beyond the complex adnexal mass. Radiographics. 2015;35(3):946-59.

3. L. LTW. Cornual (interstitial) ectopic pregnancies (IEP) in second trimester. BJOG An Int J Obstet Gynaecol. 2012;119:247.

4. Parker RA, Yano M, Tai AW, Friedman M, Narra VR, Menias CO. MR imaging findings of ectopic pregnancy: a pictorial review. Radiographics. 2012;32(5):1445-60.

5. Rizk B, Holliday CP, Abuzeid M. Challenges in the diagnosis and management of interstitial and cornual ectopic pregnancies. Middle East Fertility Society Journal. 2013;18(4):235-40.

6. Constance ES, Moravek MB. Diagnosis and Management of Ectopic Pregnancy. Handbook of Gynecology. 2017:291-304.

7. Duong D, Baker WE, Adedipe A. Clinicianperformed ultrasound diagnosis of ruptured interstitial pregnancy. The American journal of emergency medicine. 2009;27(9):1170-e1.
8. Ackerman TE, Levi CS, Dashefsky SM, Holt SC, Lindsay DJ. Interstitial line: sonographic finding in interstitial (cornual) ectopic pregnancy. Radiology. 1993;189(1):83-7.

9. Jermy K, Thomas J, Doo A, Bourne T. The conservative management of interstitial pregnancy. BJOG: An International Journal of Obstetrics \& Gynaecology. 2004;111(11):1283-8.

10. Hiersch L, Krissi H, Ashwal E, From A, Wiznitzer A, Peled Y. Effectiveness of medical treatment with methotrexate for interstitial pregnancy. Australian and New Zealand Journal of Obstetrics and Gynaecology. 2014;54(6):576-80.

11. Liao CY, Tse J, Sung SY, Chen SH, Tsui WH. Cornual wedge resection for interstitial pregnancy and postoperative outcome. Aust New Zeal J Obstet Gynaecol. 2017;57(3):342-5

12. Grindler NM, Ng J, Tocce $\mathrm{K}$, Alvero $\mathrm{R}$. Considerations for management of interstitial ectopic pregnancies: Two case reports. J Med Case Rep. 2016;10(1):106.

Cite this article as: Sharma SS, Gokhale AV, Agrawal S. A case report on ruptured interstitial ectopic pregnancy: diagnostic dilemma. Int J Reprod Contracept Obstet Gynecol 2019;8:2567-70. 